\title{
PREDICTION OF BLOOD PATTERN IN S-SHAPED MODEL OF ARTERY UNDER NORMAL BLOOD PRESSURE
}

\author{
Mohd Azrul Hisham Mohd Adib ${ }^{1}$, Norashikin Nor Tuah ${ }^{1}$ \\ Nur Hazreen Mohd Hasni ${ }^{2}$, Kahar Osman ${ }^{3}$ \\ ${ }^{1}$ Centre for Sport Engineering, \\ Faculty of Mechanical Engineering, Universiti Malaysia Pahang, \\ 26600 Pekan, Pahang, Malaysia \\ Email: azrul@ump.edu.my \\ ${ }^{2}$ Klinik Kesihatan Jaya Gading, 25150 Kuantan, Pahang, Malaysia \\ ${ }^{3}$ Faculty of Bioscience \& Medical Engineering, Universiti Teknologi Malaysia, \\ 81300 Skudai, Johor, Malaysia
}

\begin{abstract}
Athletes are susceptible to a wide variety of traumatic and non-traumatic vascular injuries to the lower limb. This paper aims to predict the three-dimensional flow pattern of blood through an S-shaped geometrical artery model. This model has created by using Fluid Structure Interaction (FSI) software. The modeling of the geometrical Sshaped artery is suitable for understanding the pattern of blood flow under constant normal blood pressure. In this study, a numerical method is used that works on the assumption that the blood is incompressible and Newtonian; thus, a laminar type of flow can be considered. The authors have compared the results with a previous study with FSI validation simulation. The validation and verification of the simulation studies is performed by comparing the maximum velocity at $\mathrm{t}=0.4 \mathrm{~s}$, because at this time, the blood accelerates rapidly. In addition, the resulting blood flow at various times, under the same boundary conditions in the S-shaped geometrical artery model, is presented. The graph shows that velocity increases linearly with time. Thus, it can be concluded that the flow of blood increases with respect to the pressure inside the body.
\end{abstract}

Keywords: Biomechanics; artery; blood flow; Fluid Structure Interaction (FSI)

\section{INTRODUCTION}

Vascular injuries are the result of traumatic and non-traumatic injury, especially to the lower limb at the knee joint. Athletes are susceptible to these types of injury because of their sporting activities. Generally, vascular injuries affect the popliteal artery most often, followed by the common femoral artery at the knee joint. Thus, patients that receive this injury will experience pain, swelling, and reduction in blood flow due to the occlusion, dissection, or aneurysm of the arteries involved. Both arterial injuries exhibit compression and interruption of flow at the knee joint (Berger, Talbot, \& Yao, 1983; Mohd Adib, Mohd Hasni, Osman, Maskon, \& Kadirgama, 2012). Thus, the flow of blood in both arteries may be affected by serious injury to the athletes during exercise, such as when cycling, running, and jumping. Both arteries are related to the blood as a working fluid in the human body, and any injury that affects these arteries has an effect on the blood flow. Numerical simulation is one method that can be applied to simulate the blood flow pattern through the main artery of the knee joint. In previous arterial research, a geometrical S-shaped artery was used for performing the investigation of the 
artery. The numerical study of nonlinear pulsatile flow in S-shaped curved arteries was undertaken by Espino, Shepherd, Hukins, and Buchan (2005). In this paper, two models of geometrical S-shaped arteries with different vessel diameters are considered. The numerical study analyzes the temporal and spatial distributions of hemodynamic variables during the cardiac cycle, such as the velocity field, secondary flow, pressure, and wall shear stresses in the arteries. A rigid tube of constant circular cross section, which is bent into an S-shape, is considered for the steady fluid flow (Baccani, Domenichini, \& Pedrizzetti, 2003; Tahseen, Ishak, \& Rahman, 2012). Athletes are vulnerable to vascular injuries of the lower limb, especially at the knee joint. From the literature, it is clear that many vascular injuries affect the main arteries of the knee, such as the popliteal and femoral arteries. Both of these arteries are very important in supplying blood around the knee joint. Retirement from sports may result if a vascular injury is received in the lower limb and if the leg loses function. A literature review reveals that traumatic arterial injuries may occur in the common femoral artery. Cycling is a popular sporting activity for many people because the sport is not too demanding. However, vascular injury may occur to the femoral artery during a fall from a bicycle. Such an occurrence is rare because the common femoral artery is generally protected by the bony pelvis and femoral sheath (Einstein, Kunzelman, Reinhall, Nicosia, \& Cochran, 2005).

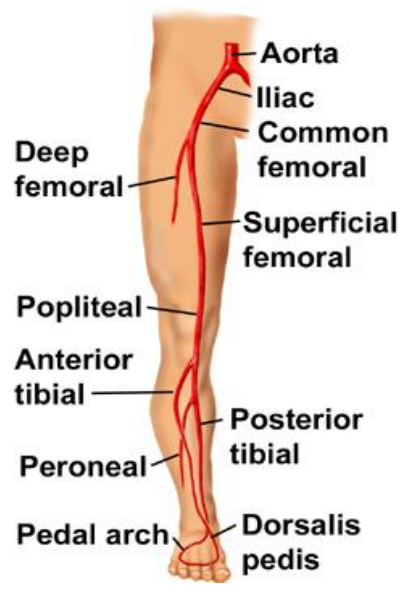

Figure 1. Femoral and popliteal artery in human leg.

Football is another activity very demanding of the lower limb, especially the knee joint. It is found in the literature that vascular injury entrapment syndrome to the popliteal artery can affect footballers. The cause of a popliteal artery injury for a footballer is blunt trauma, hypertension, dislocation, per-articular fractures, and ligament rupture. Thus, such an injury causes interruption of flow in the popliteal artery and patients may complain of pain, paresthesia, or loss of sensation or motor function distal to the knee (Berger et al., 1983; Sarfati, Galt, Treiman, \& Kraiss, 2002). In the lower limb anatomy, the main arteries for the knee joint are the popliteal and femoral arteries. The arteries of the pelvis and leg are divided by the common iliac into two branches: the internal and external iliac. The external iliac is the artery that enters the thigh and becomes the femoral artery. The femoral artery branches as it descends the thigh, one of which is the deep femoral artery. The deep femoral artery is located in the posterior thigh. This artery gives off branches that supply the head and neck femur for the lateral and medial circumflex arteries. Following that, the femoral artery descends 
and passes through the adductor hiatus and enters the popliteal fossa. Subsequently, the femoral artery becomes the popliteal artery, which is located behind the knee. The popliteal artery divides into the posterior and anterior tibial arteries. Figure 1 shows the femoral and popliteal arteries in the human leg.

\section{METHODS}

In the present numerical study, the important assumptions were made that the arteries are rigid, circular, and equi-sectional S-shaped vessels. In addition, it was assumed that the blood is an incompressible Newtonian fluid; because blood has a very complex flow, this assumption can be accepted. Finally, the authors stated that the motion of the blood in the vessels is a three-dimensional steady laminar flow.

\section{A. Geometrical model}

The geometrical model of the S-shaped vessels is shown in Figure 2. The radius of the model artery is $\mathrm{R}=0.3 \mathrm{~cm}$, taken from the paper on the human systematic circulation by Reul, Talukder, and Müller (1980). Table 1 shows that the geometrical parameter L1 is a straight vessel the length of the inlet; L2 is a straight vessel the length of the outlet; and the two bends show an angle of $45^{\circ}\left(R_{c}=3.5 \mathrm{~cm}\right)$. The geometrical model is configured for three-dimensional flow.

Table 1. Geometrical parameters of curved vessel model.

\begin{tabular}{ccccc}
\hline & $\mathrm{R}(\mathrm{cm})$ & $\mathrm{R}_{\mathrm{c}}(\mathrm{cm})$ & $\mathrm{L} 1(\mathrm{~cm})$ & $\mathrm{L} 2(\mathrm{~cm})$ \\
\hline Vessel model & 0.3 & 3.5 & 3 & 2 \\
\hline
\end{tabular}

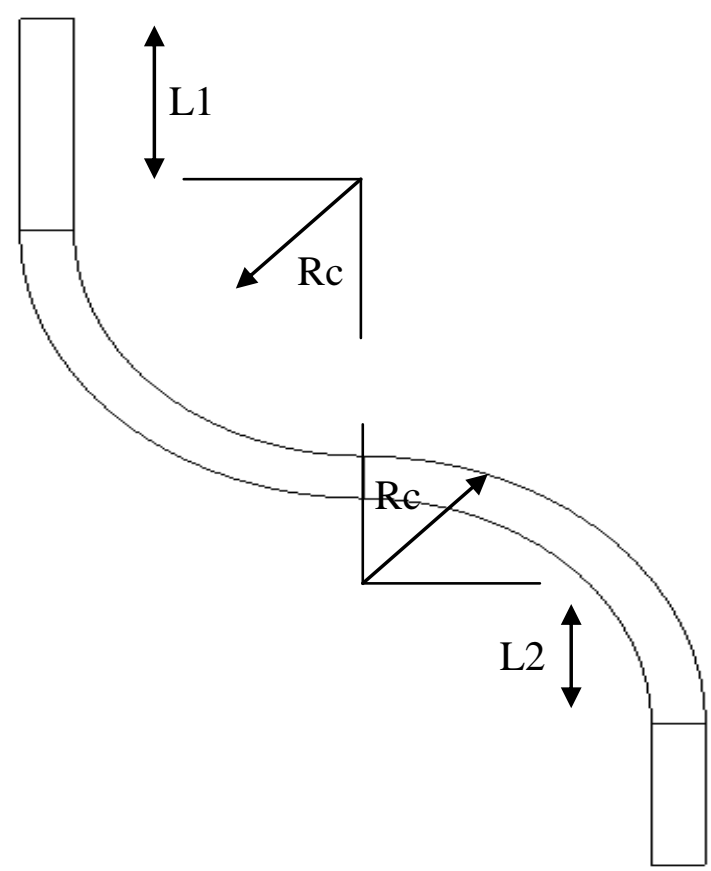

Figure 2. Geometrical model of vessels. 


\section{B. The boundary conditions}

Boundary conditions are important when applying the field of engineering to solve applications. Boundary conditions are critical parts of solving the solution encountered in the flow analyses. For this simulation in the S-shaped artery, the velocity at the inlet during exercise conditions and at the outlet is to be zero normal traction. This is because normal traction is equivalent to normal stress, which consists of pressure and normal shear stress. No slip boundary condition has been applied to the wall to prevent the blood penetrating through the wall as it flows along the artery.

\section{Parameters setup}

The parameters of the working fluid of blood are set depending on previous research by De Hart, Peters, Schreurs, and Baaijens (2003). Table 2 shows the parameters of density and viscosity for the blood. The viscosity of blood is assumed constant for simulation of the blood flow.

Table 2. Parameters setup.

\begin{tabular}{ll}
\hline Parameters & Value \\
\hline Density of blood, $\mathrm{kg} / \mathrm{m}^{3}$ & 1056 \\
Dynamical viscosity of blood, N.s $/ \mathrm{m}^{2}$ & 0.004 \\
\hline
\end{tabular}

\section{Numerical simulation method}

In this study, the geometrical S-shaped artery was considered as a rigid body. The artery model retains its original shape during the running of the simulation. The simulation investigates the blood flow pattern for constant velocity at the inlet with regard to time. The Fluid Structure Interaction (FSI) (AUI 8.5) method was used to solve the simulation of flow through the artery. The simulation of blood is unidirectional with constant normal blood pressure. The flow of blood is very complex the near the vessel wall and therefore, the meshing size must be finer in the near-wall region in order to improve the calculation process. The type of three-dimensional fluids is considered to create a mesh for the 43891 finite elements by FSI.

\section{E. Pre-setup in FSI fluid}

Figure 3 shows the flow process to set up the simulation of the fluid. First, the FSI-CFD module was opened. The flow of the 3D fluid was assumed laminar and incompressible. The material properties are constant with a given density and dynamical viscosity, as shown in Table 2. Next, the time function and time step are determined regarding the time given for every simulation. The boundary condition with no slip boundary was applied to the wall of the artery. The inlet boundary layer was applied with constant nodal forces during normal blood pressure $=120 \mathrm{mmHg}$, and at the outlet the normal zero normal traction is applied. Then, the process continued by defining the element group and by adding the type of 3D fluid. Meshing was continued after defining the element group by subdividing the density of the body. A tetrahedral-type of mesh was applied because of the number of elements of the complex flow in the S-shaped artery. 
Finally, after finishing the meshing step, the drawing could be saved into the FSI-CFD database file and the post-processing result examined.

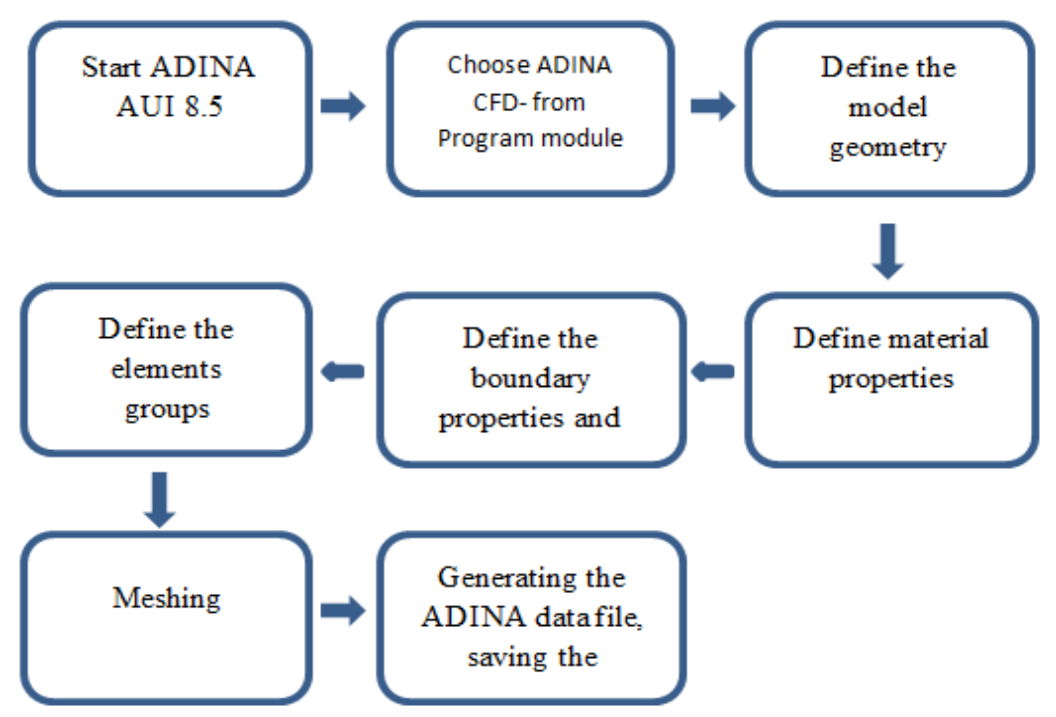

Figure 3. Flow process for FSI-Fluid setup.

\section{RESULTS AND DISCUSSION}

Figure 4 shows the comparison of the velocity between the geometrical S-shaped artery in this study's simulation and that of the model of Qiao, Guo, Wu, Zeng, and Xu (2004). Qiao et al. (2004) showed that the velocity increases linearly with time. By comparing our results with the previous study, it can be seen that velocity increases as the time is increased. However, the maximum velocity determined by Qiao et al. (2004) is higher than that obtained in the current simulation. This is because in parts of the simulation setup there is no guarantee of $100 \%$ error-free implementation. In addition, the difference may be because of the different software used for the simulation. The error between this validation study and the results of Qiao et al. (2004) is 57\%. The pressures applied to both simulation studies are within the range of normal blood pressure, which is $120 \mathrm{mmHg}$. The magnitude of maximum velocity is shown by applying the normal blood pressure through the geometrical S-shaped artery. Figure 5 shows the velocity of blood flow at various times when applying normal blood pressure. At $\mathrm{t}=0$, the blood flow is fixed to be zero velocity because there is no movement. At $t=0.04,0.072,0.12$, $0.16,0.184$, and $0.4 \mathrm{~s}$, the velocity of the blood flow increases with time. Choosing the time is done in accordance with the time of exercise activity and can be used to compare the different results for blood flow at every time selected. 


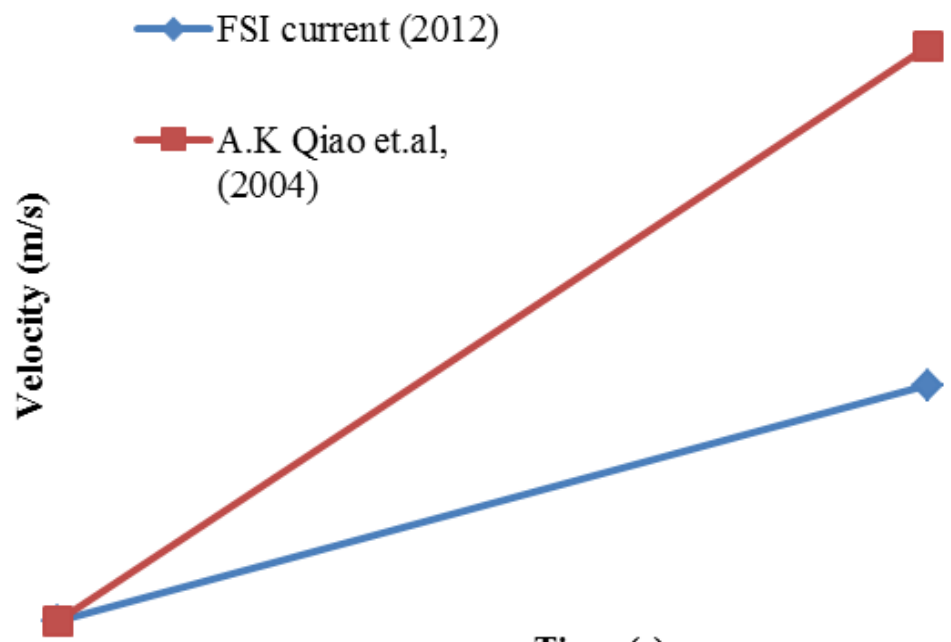

Time (s)

Figure 4. Comparison of velocity of geometrical S-shaped model.

It can be seen that the velocity of blood flow increases when applying normal blood pressure. Velocity is also dependent on blood pressure. As blood flows linearly and smoothly, the pattern is laminar because it is under normal conditions of human blood circulation. The vector pattern of blood flow is almost the same because the cross section of the geometrical S-shaped model is rigid. The working fluid of blood does not penetrate through the walls as it flows along the artery because of the no slip boundary condition on the wall, $\mathrm{v}=0$.

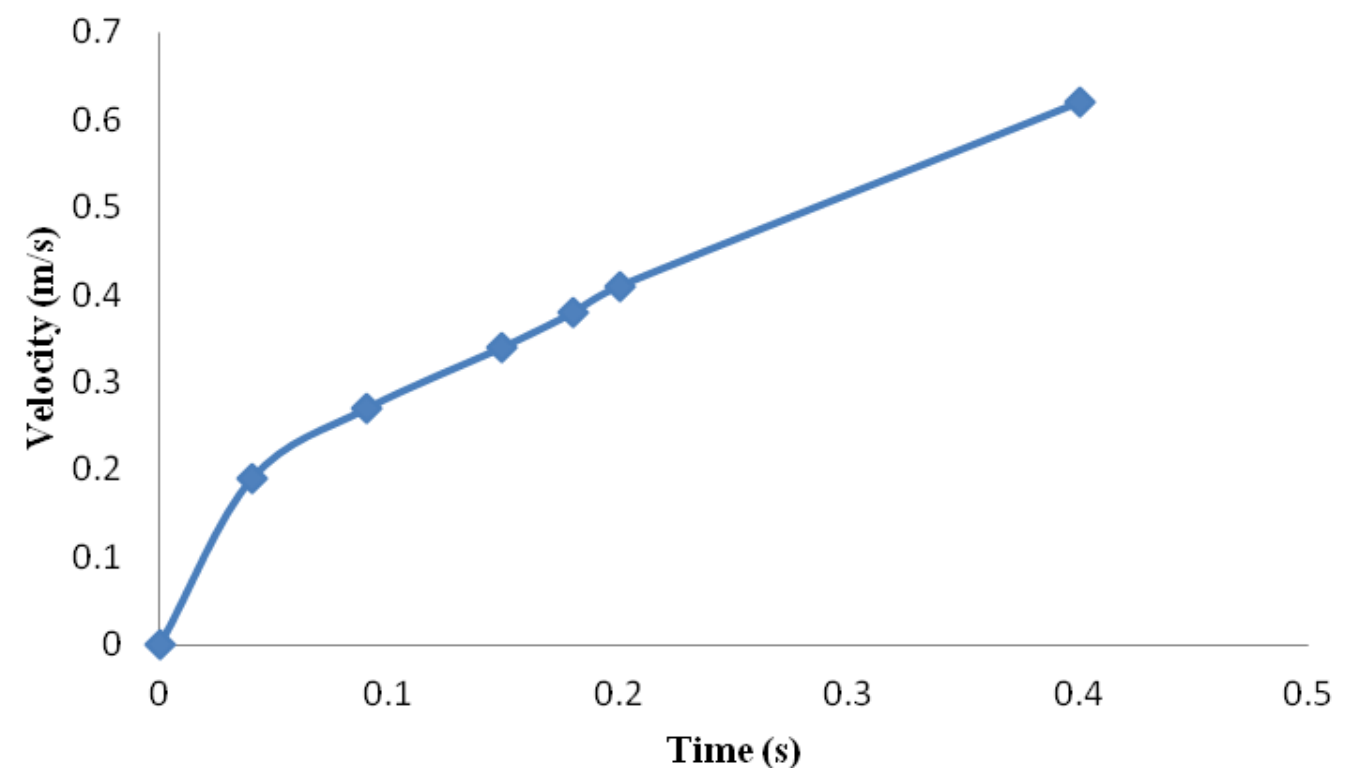

Figure 5. Velocity against time when applying normal blood pressure at $120 \mathrm{mmHg}$ 
(a)

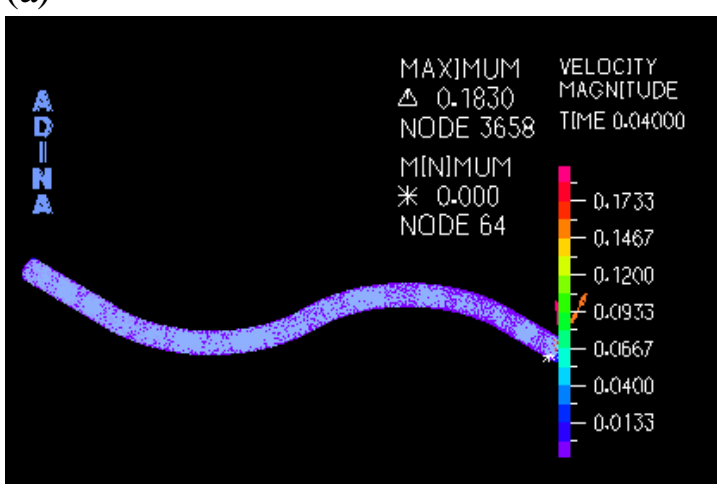

(c)

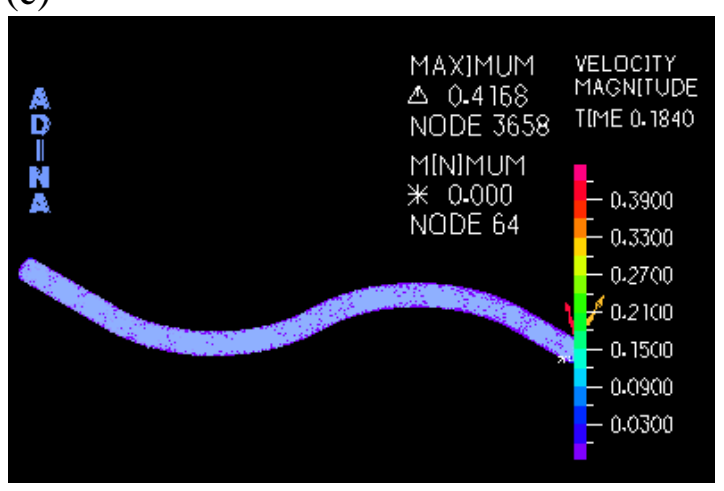

(b)

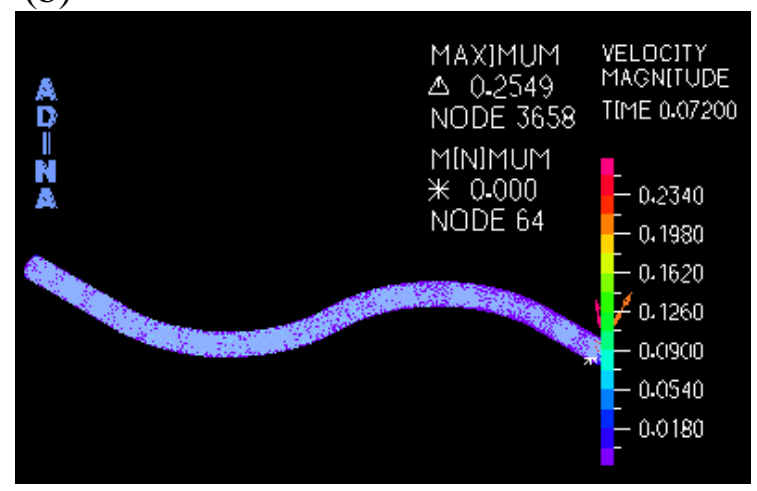

(d)

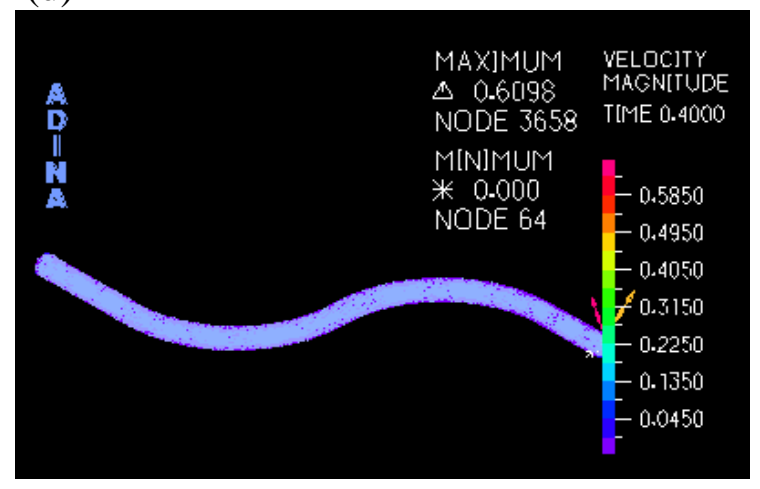

Figure 6. The maximum value of the blood flow in artery model with the same nodes at 3658. Time of blood is directly proportional with velocity of blood flow in artery model.

\section{CONCLUSION}

The prediction of 3D blood flow in an S-shaped geometrical artery, by using FSI software, has shown that the blood flow depends on the constant normal blood pressure at various times. It is shown that the velocity at the entrance accelerates rapidly until arriving at certain nodes at the maximum velocity. Thus, the simulation reveals that it is interesting to know the blood flow pattern with respect to time. In this study, the blood flow during sports activity has been considered. The velocity at different times may give different maximum velocity results at the same nodes, because the pressure of the blood in our body depends on the acceleration of the blood flow. In a circulatory system, blood pressure is highest in the arteries. Thus, during sports activities, such as cycling and running, the speed of the blood flow increases and the blood pressure in the arteries rises.

\section{ACKNOWLEDGMENTS}

The support of the Universiti Malaysia Pahang (UMP) under grant RDU110331 and of the Centre for Sport Engineering (CenSE) is gratefully acknowledged. The authors of this paper also would like to express their gratitude to Klinik Kesihatan Jaya Gading and Universiti Teknologi Malaysia (UTM) for supporting these research activities. 


\section{REFERENCES}

Baccani, B., Domenichini, F., \& Pedrizzetti, G. (2003). Model and influence of mitral valve opening during the left ventricular filling. Journal of Biomechanics, 36(3), 355-361.

Berger, S. A., Talbot. L., \& Yao, L. S. (1983). Flow in curved pipes. Annual Review of Fluid Mechanics, 15(1), 461-512.

De Hart, J., Peters, G. W. M., Schreurs, P. J. G., \& Baaijens, F. P. T. (2003). A threedimensional computational analysis of fluid-structure interaction in the aortic valve. Journal of Biomechanics, 36, 103-112.

Einstein, D. R., Kunzelman, K.S., Reinhall, P. G., Nicosia, M. A., \& Cochran, R. P. (2005). The relationship of normal and abnormal microstructural proliferation to the mitral valve closure sound. Journal of Biomechanical Engineering, 127, 134-147.

Espino, D. M., Shepherd, D. E., Hukins, D. W., \& Buchan, K. G. (2005). The role of chordae tendineae in mitral valve competence. Journal of Heart Valve Disease, 14, 603-609.

Mohd Adib, M. A. H., Mohd Hasni, N. H., Osman, K., Maskon, O., \& Kadirgama, K. (2012). Prediction of blood flow velocity and leaflet deformation via 2D mitral valve model. Journal of Mechanical Engineering and Sciences, 2. 217-225.

Qiao, A. K., Guo, K. L., Wu, S. G., Zeng, Y. J., \& Xu, X. H. (2004). Numerical study of nonlinear pulsatile flow in S-shaped curved artery. Medical Engineering and Physics, 26, 545-552.

Reul, H., Talukder, N., \& Müller, E. W. (1980). Fluid mechanics of the natural mitral valve. Journal of Biomechanics, 14, 361-372.

Sarfati, M. R., Galt, S. W., Treiman, G. S., \& Kraiss, L. W. (2002). Common femoral artery injury secondary to bicycle handlebar trauma. Journal of Vascular Surgery, 35(3), 589-591.

Tahseen, T. A., Ishak, M., \& Rahman, M. M. (2012). A numerical study of forced convection heat transfer over a series of flat tubes between parallel plates. Journal of Mechanical Engineering and Sciences, 3,271-280. 